\title{
Formação para a ética em pesquisa: um olhar para os programas de pós-graduação em Educação
}

\author{
Training for research ethics: a look at postgraduate programs in Education
}

\author{
Formación para la ética en la investigación: una mirada a los \\ programas de postgrado en Educación
}

JoÃo Batista CaRvalho Nunes*

\begin{abstract}
$\diamond$
RESUMO

A atenção dos pesquisadores brasileiros da área de Educação para a necessidade de debates, estudos e pesquisas acerca da ética em pesquisa é algo recente. O objetivo da investigação que dá suporte a este artigo é identificar, no Brasil, quais programas de pós-graduação stricto sensu da área de Educação incluem, nas ementas de suas disciplinas, o tema ética em pesquisa. Empregou-se o método da pesquisa documental, considerando-se como documentos as ementas das disciplinas dos programas de pós-graduação em Educação do Brasil, disponíveis em formato digital, na Plataforma Sucupira. Do conjunto de 8.892 disciplinas analisadas, somente $69(0,78 \%)$ incluem o tema ética em pesquisa em suas ementas. Essas disciplinas estão distribuídas por 37 programas de pós-graduação em Educação, representando $21,64 \%$ do total. Esse resultado indica a necessidade urgente de se garantir formação disciplinar e sistemática sobre o tema ética em pesquisa nos programas de pós-graduação em Educação.
\end{abstract}

Palavras-chave: Ética em pesquisa. Integridade científica. Formação de pesquisadores.

\begin{abstract}
The attention of Brazilian education researchers for the debates, studies and researches on research ethics is something recent. The objective of the research that supports this paper is to identify, in Brazil, which postgraduate programs in Education include research ethics subject in the discipline description. The documentary research method was used, considering as documents the discipline descriptions of the Brazilian postgraduate programs in Education, available in digital format in the Sucupira Platform. Of the set of 8,892 disciplines, only $69(0.78 \%)$ include the research ethics theme in their discipline description. These disciplines are distributed by 37 postgraduate programs in Education, representing 21.64\% of the total. This result indicates the urgent need to guarantee disciplinary and systematic training on the research ethics theme in postgraduate programs in Education.
\end{abstract}

Keywords: Research ethics. Scientific integrity. Researchers training.

\section{RESUMEN}

La atención de los investigadores brasileños de educación a la necesidad de debates, estudios e investigaciones sobre la ética en la investigación es algo nuevo. El objetivo de la investigación que apoya este artículo es identificar, en Brasil, que programas de postgrado en Educación incluyen el tema de la ética en la investigación en las descripciones de los cursos. Se utilizó el método de investigación documental, teniendo en cuenta como documentos las descripciones de los cursos de postgrado em Educación de Brasil, disponibles en formato digital en la Plataforma Sucupira. Del conjunto de 8.892 cursos, sólo el 69 (0,78\%) incluyen el tema de ética en la investigación en sus descripciones. Eses cursos están distribuidos en 37 programas de postgrado en Educación, lo que representa 21,64\% del total. Este resultado indica la necesidad urgente de garantizar la formación disciplinar e sistemática sobre el tema de la ética en la investigación en programas de postgrado en Educación.

Palabras clave: Ética en la investigación. Integridad científica. Formación de investigadores.

\footnotetext{
* Doutor em Filosofia e Ciências da Educação pela Universidade de Santiago de Compostela (Espanha). Professor do Programa de Pós-Graduação em Educação da Universidade Estadual do Ceará (UECE). Líder do grupo de pesquisa Laboratório de Analítica, Tecnologia Educacional e Software Livre (LATES).E-mail: <Joao.nunes@uece.br>.
} 


\section{INTRODUÇÃO}

Em 2013, a Universidade de Düsseldorf revogou o título de doutorado concedido à ministra de Educação e Pesquisa da Alemanha, Annette Schavan. Segundo concluiu o Conselho da Faculdade de Filosofia da instituição, a então ministra havia cometido plágio em sua tese, defendida em 1980 (DW, 2013). No ano anterior, a Universidade de Semmelweis, localizada em Budapeste, havia cassado o título de doutorado de Pál Schmitt, chefe de Estado da Hungria, também em virtude de plágio (DW, 2012).

No Brasil, diversos casos foram relatados, envolvendo plágio em trabalhos acadêmicos (BESSA, 2014). Para ilustrar esse fato, a Universidade Federal de Minas Gerais (UFMG) cassou, em 2015, o título de Doutor em Estudos Literários pela Faculdade de Letras de Scarleth Yone O'Hara, defendido em 2004, após constatar a existência de plágio na tese (UFMG, 2015).

Estudo envolvendo a revisão de 2.047 artigos de pesquisa nas áreas biomédica e de ciências da vida, indexados no PubMed como retratados, constatou que " $67.4 \%$ of retractions were attributable to misconduct, including fraud or suspected fraud (43.4\%), duplicate publication (14.2\%), and plagiarism (9.8\%)" (FANG; STEENC; CASADEVALL, 2012, p. 42).

Os temas de plágio, autoplágio, fabricação e falsificação de dados de pesquisa são analisados no âmbito dos estudos sobre "integridade na pesquisa" ou "integridade científica" (PITHAN; VIDAL, 2013). Devem ser considerados, todavia, como problemas éticos, pois vão de encontro às “[...] 'boas' condutas no cotidiano acadêmico, tais como a honestidade no tratamento dos dados e na divulgação do conhecimento [...]" (FARE; MACHADO; CARVALHO, 2014, p. 252). Isso revela que precisam ser contemplados quando se discute a ética em pesquisa.

Em razão do crescimento da produção científica mundial e, particularmente, da de caráter nacional (ALMEIDA; GUIMARÃES, 2013), a má conduta como pesquisador, antes, durante e depois da pesquisa, deve ser objeto de atenção constante por parte de toda a comunidade científica. Como, entretanto, as instituições formadoras de pesquisadores, particularmente os programas de pós-graduação, estão formando esses profissionais para o agir ético? Essa é uma pergunta que precisa ser respondida com a maior brevidade possível, a fim de se buscar corrigir possíveis lacunas ou distorções.

Neste artigo, contudo, a preocupação é centrada na formação de mestrandos e doutorandos da área de Educação. O objetivo da investigação que dá suporte a

\footnotetext{
“ $67,4 \%$ das retrações foram atribuídas à má conduta, incluindo fraude ou suspeita de fraude $(43,4 \%)$, duplicação de publicações $(14,2 \%)$ e plágio $(9,8 \%)$ " (traduziu-se)
}

este texto é identificar, no Brasil, quais programas de pósgraduação stricto sensu em Educação incluem, nas ementas de suas disciplinas, o tema ética em pesquisa. Este estudo amplia para todos os programas da área a investigação e as reflexões desenvolvidas em Nunes (2016).

São reconhecidos os limites que um estudo apenas centrado nas ementas das disciplinas oferece para o entendimento da formação de pesquisadores no campo da ética em pesquisa. Sustenta-se, contudo, o argumento de que, embora essa formação também possa e deva ocorrer em outros momentos formativos, como durante a orientação ou na participação de pesquisas coordenadas por pesquisadores mais experientes, o fato da existência da disciplina com o tema ética em pesquisa já sinaliza uma atenção do programa de pós-graduação com o tema.

Neste texto, cuida-se, especificamente, da formação no plano de pós-graduação stricto sensu. A educação do pesquisador, contudo, pode começar já no ensino médio e na graduação, por meio dos programas de iniciação científica. É importante atentar, portanto, para o fato de que se deve aprender o necessário posicionamento ético desde o início.

\section{FORMAÇÃO DE PESQUISADORES PARA A ÉTICA EM PESQUISA}

A atenção dos pesquisadores brasileiros da área de Educação para a necessidade de debates, estudos e pesquisas acerca da ética em pesquisa é algo recente. Apesar das críticas da área de Ciências Humanas e Sociais quanto ao modelo biomédico, expresso na Resolução $\mathrm{n}$ 196/1996 e, posteriormente, na Resolução no 466/2012, emitidas pelo Conselho Nacional de Saúde (CNS) para pesquisas envolvendo seres humanos (CARVALHO; MACHADO, 2014), a Associação Nacional de PósGraduação e Pesquisa em Educação (ANPEd) somente criou sua primeira Comissão de Ética em Pesquisa em 2015.

Nesse expediente administrativo-legal, já estava explicitado, contudo, que "as especificidades éticas das pesquisas nas ciências sociais e humanas e de outras que se utilizam de metodologias próprias dessas áreas serão contempladas em resolução complementar, dadas suas particularidades" (BRASIL, 2012, item XIII.3). Apenas em abril de 2016, após intensa atividade do GT de Ciências Humanas e Sociais² da Comissão Nacional

\footnotetext{
2 O GT de Ciências Humanas e Sociais (CHS) foi criado em julho/2013 pela CONEP para elaborar a resolução a respeito das "especificidades éticas das pesquisas nas Ciências Sociais e Humanas e de outras que se utilizam de metodologias próprias dessas áreas" (CNS, 2015). Foi composto por 18 associações nacionais de pesquisa e/ou pós-graduação das áreas de CHS, pelos representantes do CNS e do Departamento de Ciência e Tecnologia do Ministério da Saúde (DECIT/SCTIE/MS), sob coordenação de um componente da CONEP/CNS.
} 
de Ética em Pesquisa (CONEP) e do Fórum de Ciências Humanas, Sociais e Sociais Aplicadas (FCHSSA), foi estabelecida a Resolução CNS no 510/2016, cuidando de

[...] normas aplicáveis a pesquisas em Ciências Humanas e Sociais cujos procedimentos metodológicos envolvam a utilização de dados diretamente obtidos com os participantes ou de informações identificáveis ou que possam acarretar riscos maiores do que os existentes na vida cotidiana [...] (BRASIL, 2016, art. $\left.1^{\circ}\right)$.

Embora a existência de uma resolução para a seara de Ciências Humanas e Sociais deva contribuir com análises dos projetos submetidos ao Sistema CEP/CONEP mais ancoradas na especificidade dessa área, ainda permanece a busca por se criar um Conselho de Ética em Pesquisa. Este, vinculado ao Ministério da Ciência, Tecnologia, Inovações e Comunicações (MCTIC), visando a atender às pesquisas em Ciências Humanas, Sociais e Sociais Aplicadas (CARVALHO; MACHADO, 2014).

Diversas associações de pesquisadores em Educação possuem, por outro lado, os próprios códigos de ética, como, por exemplo: Australian Association for Research in Education (AARE, 1993), American Educational Research Association (AERA, 201133), British Educational Research Association (BERA, 2011) e Deutsche Gesellschaft für Erziehungswissenschaft ${ }^{4}$ (DGFE, 1999).

No Brasil, a Comissão de Ética em Pesquisa da ANPEd, como resultado do Seminário "Ética e Pesquisa em Educação", realizado nos dias 29 e 30 de setembro de 2016, está elaborando a minuta de um documento inicialmente denominado "Referências sobre Ética na Pesquisa em Educação". Outras associações de pesquisadores da área de Ciências Humanas e Sociais, como a Associação Brasileira de Antropologia (ABA) e a Sociedade Brasileira de Sociologia (SBS), já possuem seus códigos de ética.

A crescente preocupação das associações de pesquisadores e do Estado com a ética em pesquisa é motivada, entre outros aspectos, pela multiplicação de casos de plágio e autoplágio (inclusive em dissertações de mestrado e teses de doutorado), bem como pela fabricação e falsificação de dados de investigações (BESSA, 2014; MACHADO, 2012). O que exige medidas preventivas para que sejam evitados. Estudo realizado por Ison (2012, p. 234) em 100 teses de doutorado de seis universidades que ofereciam programas online, publicadas de 2009 a 2011, no serviço ProQuest Dissertation and Theses, conclui: "With $60 \%$ of dissertations having unacceptable

\footnotetext{
3 Esta foi elaborada tomando por base versões anteriores: 1992, 1996 e 2002 (AERA, 2011).

${ }^{4}$ German Educational Research Association (GERA).
}

similarity indices, this study confirms that plagiarism is in fact an issue even at the highest level of graduate education" 5 .

Em 2011, o CNPq instituiu uma Comissão Especial para propor recomendações e diretrizes sobre ética e integridade na prática científica. O relatório final recomendou "duas linhas de ação: 1) ações preventivas e pedagógicas; e 2) ações de desestímulo a más condutas, inclusive de natureza punitiva" (CNPq, 2011, p. 3). No tocante às ações preventivas e pedagógicas, foi determinado que "o CNPq deve estimular que disciplinas com conteúdo ético e de integridade de pesquisa sejam oferecidas nos cursos de pós-graduação e de graduação" (CNPq, 2011, p. 3).

A necessidade de formação dos pesquisadores em ética na pesquisa, seja mediante a realização de disciplinas que se reportem ao tema, seja por meio de seu orientador de dissertação ou tese, é ratificada por diversos autores (FARE; MACHADO; CARVALHO, 2014; GRAY; JORDAN, 2012; KALICHMAN; SWEET; PLEMMONS, 2015; KROKOSCZ, 2011; PITHAN; VIDAL, 2013). Estudo de abrangência nacional, realizado por Fisher, Fried e Feldman (2009), com 968 estudantes ou recémformados de programas de doutorado em Psicologia, nos Estados Unidos, revela que a percepção dos sujeitos investigados de estarem preparados para implementar procedimentos éticos em suas pesquisas está associada, entre outros fatores, à conclusão das disciplinas que incluem ética em pesquisa.

Além disso, Fisher, Fried e Feldman (2009) ressaltam que, segundo $60 \%$ dos respondentes, seus programas possuíam disciplina obrigatória que abordava a ética em pesquisa. Essa preocupação em formar os pesquisadores para a ética em pesquisa, por meio das disciplinas constantes nos currículos dos cursos de pós-graduação stricto sensu, também foi observada por Wyman e Henly (2015), embora ainda esteja abaixo do desejável: em seu estudo envolvendo 120 cursos de doutorado em Enfermagem, nos Estados Unidos, constataram que menos da metade $(43,33 \%)$ destes tinham ética em pesquisa/integridade científica em seus currículos.

Pesquisa desenvolvida por Nunes (2016) analisou as ementas das disciplinas dos programas de pósgraduação em Educação com os conceitos CAPES 4 e 5, da Região Nordeste do Brasil, disponíveis em formato digital na Plataforma Sucupira ${ }^{6}$. Compreendeu, portanto, somente 11 programas que ofereciam mestrado

\footnotetext{
5 "Com $60 \%$ das teses possuindo índices de similaridade inaceitáveis, esse estudo confirma que o plágio é, de fato, uma questão mesmo no mais alto nível de pós-graduação" (traduziu-se).

6 Ferramenta online para coletar informações dos programas de pósgraduação do Brasil, realizar análises e avaliações e disponibilizar informações sobre o Sistema Nacional de Pós-Graduação (SNPG).
} 
acadêmico e doutorado. Do conjunto de 876 disciplinas desses programas, somente uma, de responsabilidade do Programa de Pós-Graduação em Educação da Universidade Federal do Piauí (PPGE/UFPI), trazia o assunto ética em pesquisa. $\mathrm{O}$ estudo concluiu que havia a necessidade de os programas investirem com urgência na formação sobre ética em pesquisa.

Fortalecendo essa demanda, a Resolução CNS no 510/2016 mostra atenção à formação para o campo da ética em pesquisa na área de Ciências Humanas e Sociais, ao estabelecer, em seu art. 29, que "será instituída instância, no âmbito da Conep, [...] para a proposição de projetos de formação e capacitação na área" (BRASIL, 2016, art. 29).

\section{MetodologiA}

Foi utilizado, para responder ao objetivo da investigação, o método da pesquisa documental. Este possibilita se "compreender uma dada realidade não em sua concretização imediata, mas de forma indireta, por meio da análise de documentos produzidos pelo homem a seu respeito" (MENDES; FARIAS; NÓBREGATHERRIEN, 2011, p. 32). Nesse caso, os documentos são as ementas das disciplinas dos programas de pósgraduação em Educação do Brasil, disponíveis em formato digital na Plataforma Sucupira.

$\mathrm{O}$ estudo compreendeu três etapas. Na primeira, foi realizado o levantamento das disciplinas que possuíam, em suas ementas, a palavra "ética" na Plataforma Sucupira. Foram consultadas 8.892 disciplinas. Para tanto, abria-se cada disciplina cadastrada na Plataforma de todos os $171^{7}$ programas em funcionamento em novembro e dezembro de 2016. Em seguida, utilizava-se a ferramenta de busca do navegador para a palavra "ética". Detectado, assim, esse vocábulo, lia-se o conteúdo da ementa para se certificar da presença do termo "ética". As 277 ementas das disciplinas que satisfaziam esse critério foram separadas em um arquivo, além das informações referentes ao código e ao nome do programa de origem.

Na segunda fase, foi realizada a análise de todas as ementas, a fim de se verificar quais possuíam relação com o tema ética em pesquisa. Nessa fase, 60 ementas de disciplinas foram classificadas como atendendo claramente ao critério, enquanto 15 suscitaram dúvidas

\footnotetext{
$7 \mathrm{Na}$ época, a busca na Plataforma Sucupira por programas na área de avaliação "Educação" oferecia como resultado 173 programas. Análise detalhada mostrou, contudo, que dois eram em rede: Mestrado Acadêmico em Educação da Universidade Estadual de Roraima (UERR) e do Instituto Federal de Educação, Ciência e Tecnologia de Roraima (IFRR); e Mestrado Acadêmico em Educação, Culturas e Identidades da Universidade Federal Rural de Pernambuco (UFRPE) e da Fundação Joaquim Nabuco (FJN). Como nesses dois casos apenas um programa é executado por duas instituições, o levantamento abrangeu 171 programas.
}

quanto à sua vinculação ao tema ética em pesquisa. As disciplinas desconsideradas nesse momento traziam a ética relacionada a assuntos como Direitos Humanos, Educação Ambiental, Filosofia etc.

Nesse momento, também foram encontradas oito disciplinas em seis programas com nome e ementas iguais, denominações idênticas, mas ementas diferentes, além de intitulações variadas e ementas iguais. Como os dados constantes na Plataforma Sucupira são informados pelos respectivos programas de pós-graduação, decidiu-se não se eliminar nenhuma disciplina, haja vista não se conhecer a causa que os levou a realizar esse tipo de cadastro.

A terceira fase se constituiu da segunda análise, circunscrita às 15 ementas anteriormente citadas. Para auxiliar no esclarecimento da relação ou não da ementa com o tema ética em pesquisa, foram demandadas informações complementares sobre o enfoque e sobre a natureza das respectivas disciplinas nos sites dos programas e nas referências bibliográficas das disciplinas informadas na Plataforma Sucupira. Somente nove ementas foram consideradas como atendendo ao critério proposto. Ao final, obteve-se o total de 69 ementas das disciplinas dos programas de pós-graduação em Educação do Brasil que incluem o tema ética em pesquisa.

Os dados quantitativos referentes às ementas das disciplinas dos programas foram registrados, ao longo das fases, em uma planilha eletrônica, incluindo a quantidade total de ementas e daquelas que atendiam ao critério, além de informações dos programas, como unidade federada e região onde está localizado, modalidade (acadêmico/ profissional), regime (público/privado) e nível de formação (mestrado acadêmico/mestrado acadêmico e doutorado/mestrado profissional).

Essa planilha foi importada para o software de análise estatística Stata (versão 14.2). Com base nos dados, foram produzidos gráficos e tabelas, assim como realizado o cálculo de estatísticas descritivas e de associação, de modo a oferecer subsídios para a análise do fenômeno sob exame (DANCEY; REIDY, 2013; FÁVERO et al., 2014).

\section{Discussão dos RESUlTAdos}

Em dezembro de 2016, existiam 171 programas de pós-graduação em funcionamento, cuja área de avaliação na CAPES era "Educação". Esses programas compreendiam 128 mestrados acadêmicos (74,85\%), 43 mestrados profissionais $(25,15 \%)$ e 74 doutorados $(43,27 \%)$. Havia, portanto, 54 programas que ofereciam apenas mestrado acadêmico e 74 que possuíam mestrado acadêmico e doutorado.

Esses programas estão assim distribuídos pelas cinco regiões do Brasil: 40,35\% (69) no Sudeste, 24,56\% (42) 
no Sul, $18,71 \%$ (32) no Nordeste, 9,39\% (16) no CentroOeste e 7,02\% (12) no Norte. Sudeste e Sul aglutinam, por conseguinte, mais da metade $(64,91 \%)$ dos programas de pós-graduação em Educação.

No tocante ao conceito atribuído pela CAPES, observa-se concentração de $84,21 \%$ nos estratos mais baixos ( 3 e 4), enquanto no conceito 3 estão 91 programas $(53,22 \%)$, ou seja, mais da metade; no conceito 4, encontram-se 53 programas (30,99\%). Conjunto pequeno de programas divide os estratos superiores: 18 (10,53\%) possuem conceito 5 , seis $(3,51 \%)$ estão no conceito 6 e somente três $(1,75 \%)$ alcançaram o conceito 7 .

Os 171 programas investigados totalizaram 8.892 disciplinas cadastradas na Plataforma Sucupira, com média aritmética de 52 disciplinas e desvio padrão de 62,35 disciplinas. Essa expressiva variação decorre da grande amplitude: enquanto o programa com menor número de disciplinas possui apenas dez, o de maior número tem 620. Metade dos programas, contudo, informam até 34 disciplinas. A quantidade das disciplinas mais frequente é 12, valor encontrado em oito programas. No Gráfico 1, é possível visualizar algumas dessas medidas, assim como a existência de valores atípicos (outliers).

Gráfico 1. Box plot da quantidade total das disciplinas dos programas de pós-graduação em Educação do Brasil - dez./2016

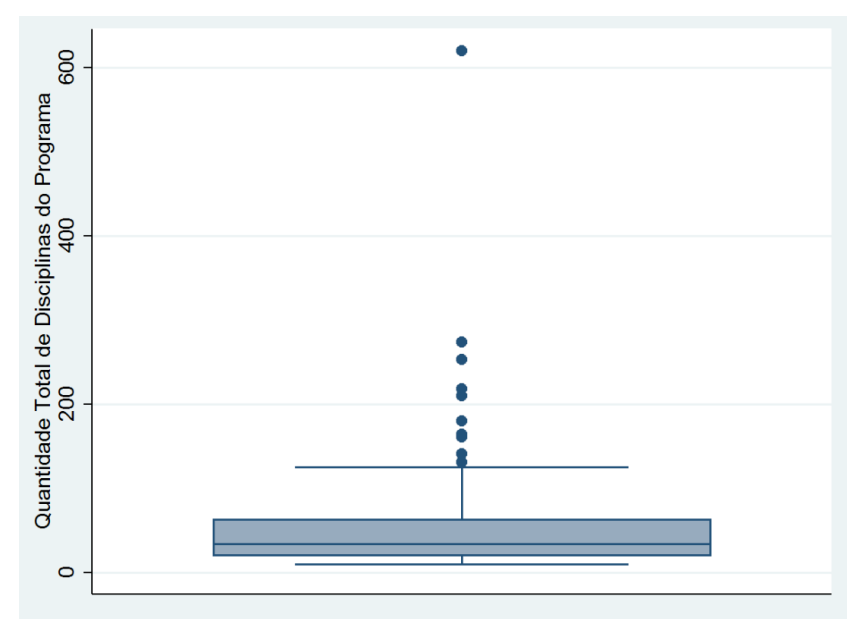

(Fonte: Elaboração própria, 2017)

A área de Educação conta, conforme se pode depreender dos dados expressos, com um número considerável de programas de pós-graduação stricto sensu no Brasil e com uma história iniciada há mais de 50 anos, quando da criação, no Brasil, do primeiro curso de mestrado em Educação, na Pontifícia Universidade Católica do Rio de Janeiro - PUC/RJ (FÁVERO, 2005). Os resultados obtidos, no entanto, mostram quão incipiente ainda está essa área no tocante à formação disciplinar no campo da ética em pesquisa.
Do conjunto das disciplinas analisadas, somente 69 $(0,78 \%)$ delas incluem o tema ética em pesquisa em suas ementas (Tabela 1). Essas disciplinas estão distribuídas por 37 programas de pós-graduação em Educação, representando $21,64 \%$ do total. A média aritmética das disciplinas que trazem a ética em pesquisa é 1,86 , com desvio padrão de 2,06 disciplinas. Mais de 2/3 desses programas $(70,27 \%)$ possuem somente uma disciplina que traz o tema ética em pesquisa em sua ementa. Esse valor de uma disciplina é, simultaneamente, mediana e moda desse conjunto de dados. O Gráfico 2 torna evidente a maneira como programas contendo mais de três disciplinas são casos atípicos.

Tabela 1. Distribuição das disciplinas de programas de pós-graduação em Educação do Brasil que incluem o tema ética em pesquisa - dez./2016

\begin{tabular}{cccc}
\hline $\begin{array}{c}\text { Disciplinas com } \\
\text { Ética em Pesquisa }\end{array}$ & Freq. & \% & Cum. \\
\hline 1 & 25 & 70.27 & 70.27 \\
2 & 5 & 13.51 & 83.78 \\
3 & 2 & 5.41 & 89.19 \\
4 & 2 & 5.41 & 94.59 \\
8 & 1 & 2.70 & 97.30 \\
11 & 1 & 2.70 & 100.00 \\
\hline Total & 37 & 100.00 & \\
\hline
\end{tabular}

(Fonte: Elaboração própria, 2017)

Gráfico 2. Box plot da quantidade das disciplinas dos programas de pós-graduação em Educação do Brasil que incluem o tema ética em pesquisa - dez./2016

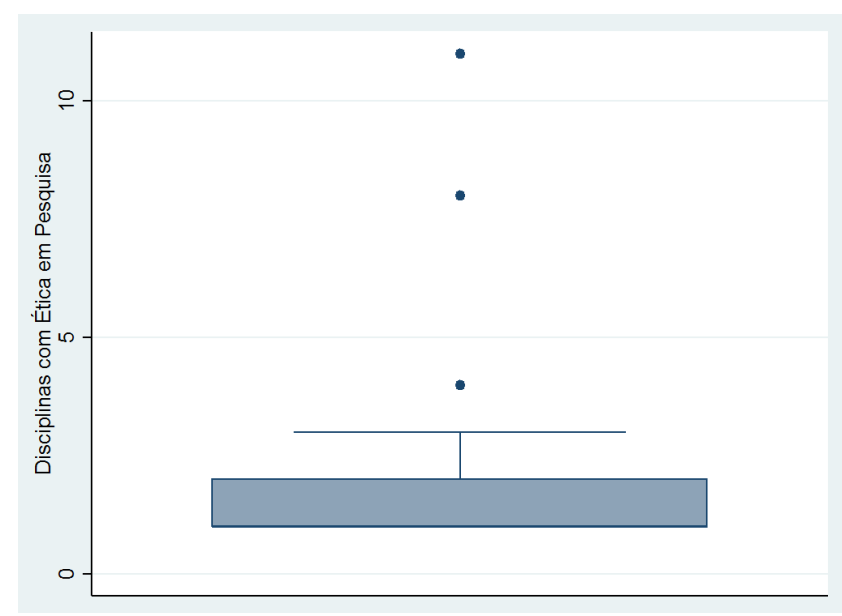

(Fonte: Elaboração própria, 2017)

O programa com o maior número de disciplinas (11) é o da Universidade Estadual de Ponta Grossa (UEPG). Deve-se frisar o fato de que esse programa é responsável pelo periódico Práxis Educativa (ISSN 1809-4309), que 
publicou uma seção temática sobre ética na pesquisa em 2014 (v. 9, n. 1). Tal seção foi apresentada pelo então coordenador do programa e representante da ANPEd no GT de Ciências Sociais e Humanas da CONEP. A situação conduz a um interrogante: o fato de o programa ter como coordenador um pesquisador que participava das discussões sobre ética em pesquisa influencia a oferta das disciplinas que incluem esse tema? Esta é uma pergunta a ser respondida por outras pesquisas.

A análise do nome das disciplinas e do conteúdo das ementas revela que estas versam sobre metodologia da pesquisa educacional e/ou redação científica. Nenhuma delas cuida apenas da ética em pesquisa. Esse tema é um entre outros abordados pelas disciplinas. Para ilustrar esse fato, destaca-se a ementa da disciplina Seminário de Tese I, do Programa de Pós-Graduação em Educação da UEPG:

Discussão do projeto de tese, bem como aprofundamento das abordagens e dos procedimentos de pesquisa. Discussão de questões teórico-metodológicas da pesquisa em educação a partir das temáticas de pesquisa dos doutorandos. Conteúdo programático: 1 - Relevância e limitações das pesquisas em educação; 2 - As condições empírico-metodológicas de uma tese; 3 - Cruzamento transversal de teses e outras produções de pesquisas com os elementos do projeto; 4 - Determinação do foco empírico-metodológico inovador num projeto de tese; 5 -Procedimentos éticos e protocolos de ética na pesquisa; 6 - Revisão e reestruturação do projeto apresentado no Processo seletivo; Avaliação: Relatório circunstanciado dos avanços e alterações do Projeto de Pesquisa (CAPES, 2016 - grifo do autor)

Embora os percentuais das disciplinas cujas ementas trazem o tema ética em pesquisa $(0,78 \%)$ e de programas que as ofertam $(21,64 \%)$ ainda sejam pequenos, são notoriamente superiores aos encontrados por Nunes (2016) para programas que ofereciam, simultaneamente, mestrado acadêmico e doutorado na Região Nordeste: $0,11 \%$ e $9,09 \%{ }^{8}$, respectivamente. Em ambos os casos, o resultado está muito distante da situação descrita por Wyman e Henly (2015) e Fisher, Fried e Feldman (2009), respetivamente, para as áreas de Enfermagem e Psicologia, nos Estados Unidos.

Tais achados devem, ademais, ser considerados com cautela. $\mathrm{O}$ fato de o tema ética em pesquisa somente aparecer em 69 disciplinas entre as analisadas não significa que este não seja desenvolvido em outros programas, além dos 37 encontrados. É possível que seja incluído

\footnotetext{
8 O autor não fez esse cálculo, mas isso pode ser facilmente deduzido, pois somente foi encontrado um programa com disciplina que incluía o tema ética em pesquisa de um total de 11 investigados.
}

no programa das disciplinas, malgrado não constar na ementa $^{9}$, ou ser ensinado durante o acompanhamento realizado pelo orientador da dissertação ou tese, ou por meio do exemplo desse orientador no desenvolvimento de suas pesquisas (GRAY; JORDAN, 2012). O resultado alerta, todavia, para a situação que exige dos programas, o mais brevemente possível, a assunção da responsabilidade pela formação sistematizada sobre ética em pesquisa.

As disciplinas que incluem o tema ética em pesquisa são oferecidas por programas distribuídos nas cinco regiões brasileiras (Gráfico 3). Diferentemente do que ocorre com o conjunto dos programas de pós-graduação em Educação do Brasil, a Região Sul possui o maior número de programas (16 ou 43,24\%), seguida pelo Sudeste (dez ou 27,03\%). As regiões Nordeste e CentroOeste empatam na quantidade de programas, isto é, cinco $(13,51 \%)$ para cada uma, enquanto o Norte só tem um programa $(2,70 \%)$.

Gráfico 3. Distribuição dos programas de pós-graduação em Educação do Brasil com disciplinas que incluem o tema ética em pesquisa, conforme as regiões - dez./2016

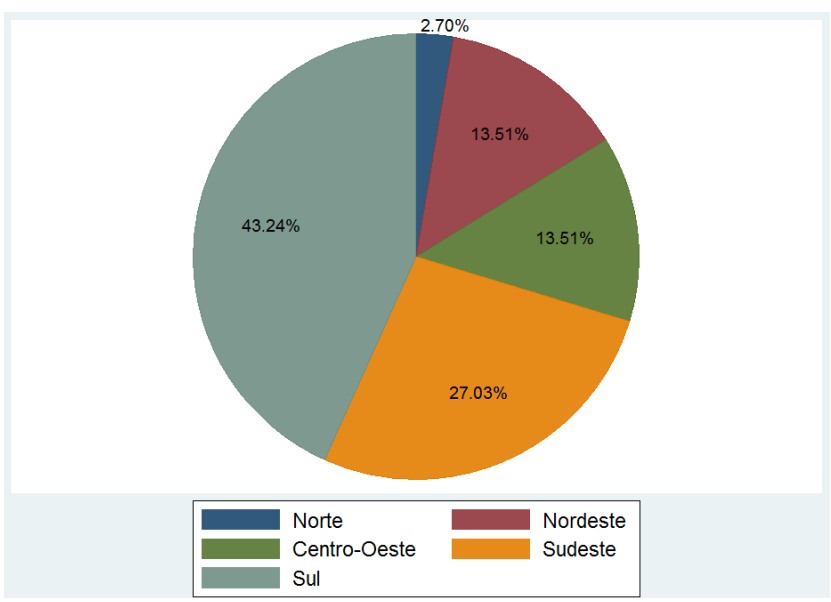

(Fonte: Elaboração própria, 2017)

Embora ocorram nas cinco regiões do Brasil, as disciplinas que incluem o tema ética em pesquisa estão em apenas $15(55,55 \%)$ dos 26 estados mais o Distrito Federal (Tabela 2). O estado com a maior quantidade de programas que as oferece é o Rio Grande do Sul, possuindo sete programas. Sete estados possuem somente um programa: Espírito Santo, Goiás, Minas Gerais, Pernambuco, Piauí, Rio Grande do Norte e Roraima.

\footnotetext{
9 Por exemplo, a disciplina que se ministra na Universidade Estadual do Ceará - UECE, intitulada "Seminário de Prática de Pesquisa I: Bases Epistemológicas da Pesquisa em Educação", do Programa de Pós-Graduação em Educação dessa academia, possui uma unidade sobre "ética na investigação em Educação", embora não conste na sua ementa.
} 
Tabela 2. Distribuição dos programas de pós-graduação em Educação do Brasil com disciplinas que incluem o tema ética em pesquisa, por unidades federativas - dez./2016

\begin{tabular}{lccr}
\hline UF & Freq. & \% & Cum. \\
\hline DF & 2 & 5.41 & 5.41 \\
ES & 1 & 2.70 & 8.11 \\
GO & 1 & 2.70 & 10.81 \\
MG & 1 & 2.70 & 13.51 \\
MT & 2 & 5.41 & 18.92 \\
PB & 2 & 5.41 & 24.32 \\
PE & 1 & 2.70 & 27.03 \\
PI & 1 & 2.70 & 29.73 \\
PR & 3 & 8.11 & 37.84 \\
RJ & 4 & 10.81 & 48.65 \\
RN & 1 & 2.70 & 51.35 \\
RR & 1 & 2.70 & 54.95 \\
RS & 7 & 18.92 & 72.97 \\
SC & 6 & 16.22 & 89.19 \\
SP & 4 & 10.81 & 100.00 \\
\hline Total & 37 & 100.00 & \\
\hline
\end{tabular}

(Fonte: Elaboração própria, 2017)

Tabela 3. Distribuição dos programas de pós-graduação em Educação do Brasil com disciplinas que incluem o tema ética em pesquisa, por IES - dez./2016

\begin{tabular}{lccr}
\hline \multicolumn{1}{c}{ IES } & Freq. & \% & Cum. \\
\hline UERJ & 3 & 8.11 & 8.11 \\
UFFS & 2 & 5.41 & 18.51 \\
UFMT & 2 & 5.41 & 18.92 \\
CEETEPS & 1 & 2.70 & 21.61 \\
FUFPI & 1 & 2.70 & 24.32 \\
FURB & 1 & 2.70 & 27.03 \\
PUC/PR & 1 & 2.70 & 29.73 \\
PUC/SP & 1 & 2.70 & 32.43 \\
UCB & 1 & 2.70 & 35.14 \\
UEPB & 1 & 2.70 & 37.84 \\
UEPG & 1 & 2.70 & 40.54 \\
UERR & 1 & 2.70 & 43.24 \\
UFCG & 1 & 2.70 & 45.95 \\
UFES & 1 & 2.70 & 48.65 \\
UFG & 1 & 2.70 & 51.35 \\
UFPEL & 1 & 2.70 & 54.05 \\
UFRGS & 1 & 2.70 & 56.76 \\
UFRN & 1 & 2.70 & 59.46 \\
UFRRJ & 1 & 2.70 & 62.16 \\
UFSC & 1 & 2.70 & 64.85 \\
UNB & 1 & 2.70 & 67.57 \\
UNIARP & 1 & 2.70 & 70.27 \\
UNICID & 1 & 2.70 & 72.97 \\
UNIJUÍ & 1 & 2.70 & 75.68 \\
UNIOESTE & 1 & 2.70 & 78.38 \\
UNISAL & 1 & 2.70 & 81.08 \\
UNISC & 1 & 2.70 & 83.78 \\
UNISINOS & 1 & 2.70 & 86.49 \\
UNIUBE & 1 & 2.70 & 89.19 \\
UNIVALI & 1 & 2.70 & 91.89 \\
UNIVILLE & 1 & 2.70 & 94.59 \\
UPE & 1 & 2.70 & 97.30 \\
URI & 1 & 2.70 & 100.00 \\
\hline Total & 100.00 & \\
\hline & 1 & & \\
\hline & 1 & 1 & \\
\hline
\end{tabular}

(Fonte: Elaboração própria, 2017)
Os 37 programas são ofertados por 33 instituições de educação superior - IES (Tabela 3). Observa-se que, em três, há mais de um programa de pós-graduação em Educação com disciplina que inclui o tema ética em pesquisa: Universidade do Estado do Rio de Janeiro (UERJ), Universidade Federal da Fronteira do Sul (UFFS) e Universidade Federal de Mato Grosso (UFMT). Nas demais, apenas existe um programa. Quase 2/3 $(62,16 \%)$ dos programas estão localizados em instituições públicas, enquanto o restante (37,84\%) está em academias particulares. Dos 23 programas de IES públicas, 14 $(60,87 \%)$ são de instituições federais e $9(39,13 \%)$ de estaduais.

Com uma distribuição próxima à apresentada pelo conjunto dos programas de pós-graduação em Educação do Brasil, os 37 programas com disciplinas que incluem o tema ética em pesquisa compreendiam 30 mestrados acadêmicos $(81,08 \%)$, sete mestrados profissionais $(18,82 \%)$ e 19 doutorados $(51,35 \%)$. Havia, por conseguinte, 11 programas que ofereciam somente mestrado acadêmico e 19 que possuíam mestrado acadêmico e doutorado.

Em relação ao conceito dos programas atribuído pela CAPES, a situação também é semelhante ao que ocorre no conjunto da pós-graduação em Educação brasileira. Mais de $80 \%$ dos programas que ofertam disciplinas com ementa incluindo ética em pesquisa estão nos estratos mais baixos (3 e 4). Nesses programas, no entanto, há uma distribuição igualitária entre os conceitos 3 e 4: há 15 programas $(40,54 \%)$ com cada um dos conceitos. Os demais estão nos estratos superiores: cinco $(13,51 \%)$ possuem o conceito 5 e cada um dos conceitos 6 e 7 tem um programa representado $(2,70 \%)$.

\section{CONSIDERAÇõES FINAIS}

Esta investigação revela que os programas de pósgraduação em Educação do Brasil, malgrado o crescimento do debate na área sobre ética em pesquisa, carecem de disciplinas que abordem esse tema. Do conjunto de 8.892 analisadas, somente $69(0,78 \%)$ incluem o tema ética em pesquisa em suas ementas. Essas disciplinas estão distribuídas por 37 programas de pós-graduação em Educação, representando 21,64\% do total. Se o programa não oferecer outros mecanismos para a formação de seus mestrandos e doutorandos nesse tema, pode-se estar comprometendo a qualidade e a confiabilidade das pesquisas e da própria formação das futuras gerações de pesquisadores da área de Educação.

Tal circunstância exige algumas medidas, com urgência. De um lado, é preciso que os programas de pósgraduação definam, no mínimo, uma disciplina sobre ética em pesquisa, ou insiram esse tema como conteúdo de uma 
disciplina da área da metodologia da pesquisa educacional. Esta, contudo, não pode versar somente sobre os aspectos normatizados pelas Resoluções CNS no 466/2012 e no 510/2016, que se limitam à relação entre pesquisador e participante da pesquisa. É preciso conceber que a prática profissional do pesquisador deve ser conduzida de maneira ética, não se limitando ao processo de investigação. Deve abranger também a publicação científica, a participação em eventos e a avaliação de trabalhos científicos (bancas de qualificação e defesa, comissões de assessoramento a agências de fomento, comitês editoriais de periódicos, comissões científicas de eventos etc.), além do próprio exercício da docência e da gestão acadêmica. Em síntese, o estudo da ética em pesquisa precisa tratar sobre o antes, o durante e o depois da investigação.

De outro lado, os programas não podem olvidar de seus próprios professores. Dado o acelerado desenvolvimento científico e tecnológico, com novos desafios à privacidade e à autoria em um mundo cada vez mais digital, é necessário também pensar sobre a formação do corpo docente no tema ética em pesquisa. Os exemplos e orientações dos professores poderão influenciar os estudantes sob sua supervisão, indicando modelos de conduta na pesquisa. Palestras, seminários, oficinas e demais tipos de eventos na área, além da constituição de uma rede virtual de apoio para discussão sobre o tema, podem ser usados para formar os professores dos programas, assim como mestrandos e doutorandos.

Neste momento em que a área de Educação assume o desafio de pensar referenciais específicos para a ética em pesquisa, torna-se mais relevante a ideia de que todos os pesquisadores e futuros investigadores busquem se assenhorear das discussões, avanços e práticas do campo da ética em pesquisa.

\section{REFERÊNCIAS}

ALMEIDA, E. C. E.; GUIMARÃES, J. A. A pós-graduação e a evolução da produção científica brasileira. São Paulo: Editora Senac São Paulo, 2013.

AMERICAN EDUCATIONAL RESEARCH ASSOCIATION (AERA). Code of ethics. Educational Researcher, v. 40, n. 3, p. 145-156, 2011.

AUSTRALIAN ASSOCIATION FOR RESEARCH IN EDUCATION (AARE). Code of ethics. Deakin: AARE, 1993. Disponível em: <http://www.aare.edu.au/pages/aare-code-ofethics.html>. Acesso em: 8 jul. 2016.

BESSA, J. C. R. Por uma cultura de ética e de integridade do pesquisador em formação inicial. Revista Espaço Acadêmico, n. 159, p. 114-121, 2014.

BRASIL. Conselho Nacional de Saúde. Resolução no 466, de 12 de dezembro de 2012. Brasília: CNS, 2012.

BRASIL. Conselho Nacional de Saúde. Resolução no 510, de 7 de abril de 2016. Brasília: CNS, 2016.
BRITISH EDUCATIONAL RESEARCH ASSOCIATION (BERA). Ethical guidelines for educational research. London: BERA, 2011.

CARVALHO, I. C. M.; MACHADO, F. V. A regulação da pesquisa e o campo biomédico: considerações sobre um embate epistêmico desde o campo da educação. Práxis Educativa, v. 9, n. 1, p. 209-234, 2014.

CONSELHO NACIONAL DE DESENVOLVIMENTO CIENTÍFICO E TECNOLÓGICO (CNPQ). Relatório da Comissão de Integridade de Pesquisa do CNPq. Brasília: CNPq, 2011.

CONSELHO NACIONAL DE SAÚDE (CNS). Desenvolvido pela Equipe de Comunicação do CNS, 2015. Apresenta consulta à sociedade de Minuta de Resolução Complementar sobre "As especificidades éticas das pesquisas nas Ciências Sociais e Humanas e de outras que se utilizam de metodologias próprias dessas áreas". Disponível em: <http://conselho.saude.gov.br/ ultimas_noticias/2015/07jul21_minuta.html>. Acesso em: 8 jul. 2015.

COORDENAÇÃO DE APERFEIÇOAMENTO DE PESSOAL DE NÍVEL SUPERIOR (CAPES). Plataforma Sucupira. Brasília, 2016. Disponível em: <https://sucupira.capes.gov. br/sucupira/public/consultas/coleta/disciplina/viewDisciplina. jsf?popup=true\&id_disciplina=122499>. Acesso em: 30 nov. 2016.

DANCEY, C. P.; REIDY, J. Estatística sem Matemática para Psicologia. 5. ed. Porto Alegre: Penso, 2013.

DEUTSCHE GESELLSCHAFT FÜR ERZIEHUNGSWISSENSCHAFT (DGFE). Code of ethics of Deutsche Gesellschaft für Erziehungswissenschaft (DGfE). Berlin: DGfE, 1999.

DW. Presidente da Hungria renuncia devido a escândalo de plágio. Deutsche Welle, Bonn (Alemanha), 2 abr. 2012. Disponível em: $<\mathrm{http}: / / \mathrm{dw} . c 0 \mathrm{~m} / \mathrm{p} / 14 \mathrm{WcT}>$. Acesso em: 20 fev. 2017.

DW. Universidade revoga título de doutorado da ministra alemã da Educação. Deutsche Welle, Bonn (Alemanha), 5 fev. 2013. Disponível em: $<\mathrm{http}: / / \mathrm{dw} . c 0 \mathrm{~m} / \mathrm{p} / 17 \mathrm{YcK}>$. Acesso em: $20 \mathrm{fev}$. 2017.

FANG, F. C.; STEENC, R. G.; CASADEVALL, A. Misconduct accounts for the majority of retracted scientific publications. PNAS, v. 109, n. 42, p. 17028-17033, 2012.

FARE, M.; MACHADO, F. V.; CARVALHO, I. C. M. Breve revisão sobre regulação da ética em pesquisa: subsídios para pensar a pesquisa em educação no Brasil. Práxis Educativa, v. 9, n. 1, p. 247-283, 2014.

FÁVERO, L. P.; BELFIORE, P.; TAKAMATSU, R. T.; SUZART, J. Métodos quantitativos com Stata. Rio de Janeiro: Elsevier, 2014.

FÁVERO, M. L. A. Durmeval Trigueiro Mendes e sua contribuição à pós-graduação em educação. Revista Brasileira de Educação, n. 30, p. 36-46, 2005.

FISHER, C. B.; FRIED, A. L.; FELDMAN, L. G. Graduate socialization in the Responsible Conduct of Research: a national survey on the research ethics training experiences of psychology doctoral students. Ethics \& Behavior, v. 19, n. 6, p. 496-518, 2009. 
GRAY, P. W.; JORDAN, S. R. Supervisors and academic integrity: supervisors as exemplars and mentors. J Acad Ethics, v. 10, p. 299-311, 2012.

ISON, D. C. Plagiarism among dissertations: prevalence at online institutions. J Acad Ethics, v. 10, p. 227-236, 2012.

KALICHMAN, M.; SWEET, M.; PLEMMONS, D. Standards of sientific conduct: disciplinary differences. Sci Eng Ethics, v. 21, p. 1085-1093, 2015.

KROKOSCZ, M. Abordagem do plágio nas três melhores universidades de cada um dos cinco continentes e do Brasil. Revista Brasileira de Educação, v. 16, n. 48, p. 745-818, 2011.

MACHADO, U. Chalita fez autoplágio para obter mestrado. Folha de S. Paulo, São Paulo, 12. set. 2012. Disponível em: $<$ http://www1.folha.uol.com.br/fsp/poder/25452-chalita-fezautoplagio-para-obter-mestrado.shtml>. Acesso em: 8 jul. 2016.

MENDES, E. T. B.; FARIAS, I. M. S.; NÓBREGATHERRIEN, S. M. Trabalhando com materiais diversos e exercitando o domínio da leitura: a pesquisa bibliográfica e a pesquisa documental. In: NÓBREGA-THERRIEN, S. M.; FARIAS, I. M. S.; NUNES, J. B. C. Pesquisa científica para iniciantes: caminhando no labirinto. v. 3. Fortaleza: EdUECE, 2011. p. 25-42.
NUNES, J. B. C. Formação de pesquisadores em Educação para a ética em pesquisa. In: CARVALHO, M. V. C.; CARVAlHÊdO, J. L. P.; ARAÚJO, F. A. M. (Org.). Caminhos da pós-graduação em Educação no Nordeste do Brasil: avaliação, financiamento, redes e produção científica. Teresina: EDUFPI, 2016. p. 59-71.

PITHAN, L. H.; VIDAL, T. R. A. O plágio acadêmico como um problema ético, jurídico e pedagógico. Direito \& Justiça, v. 39, n. 1, p. 77-82, 2013.

UNIVERSIDADE FEDERAL DE MINAS GERAIS (UFMG). UFMG comprova plágio e cassa título de doutora de exestudante da Faculdade de Letras. Agência de Notícias, Belo Horizonte, 27 out. 2015. Disponível em: <https://www. ufmg.br/online/arquivos/040650.shtml>. Acesso em: 20 fev. 2017.

WYMAN, J. F.; HENLY, S. J. PhD programs in nursing in the United States: visibility of American Association of Colleges of Nursing core curricular elements and emerging areas of science. Nursing Outlook, v. 63, p. 390-397, 2015.

Recebido em 16-03-2017.

Aprovado em 18-05-2017. 